\title{
Conocimientos, actitudes y prácticas de prevención sobre la enfermedad renal crónica en diabéticos e hipertensos
}

\author{
DOI 10.5377/alerta.v4i1.9538 \\ Teresa del Rosario Fuentes Martínez ${ }^{1}$ \\ Nely del Carmen Ríos ${ }^{2}$ \\ José Benjamín Argueta Coca ${ }^{3}$ \\ 1. Región Oriental de Salud, Ministerio de Salud, San Miguel, El Salvador. \\ 2. Unidad Comunitaria de Salud Familiar Cacaopera, Ministerio de Salud, Morazán, El Salvador \\ 3. SIBASI Morazán. Región Oriental de Salud, Ministerio de Salud \\ ${ }^{*}$ Correspondencia \\ $\square$ trfuentes78@gmail.com \\ (D) 0000-0002-7985-1064
}

G

ACCESO ABIERTO

Knowledge, attitudes and prevention practices of chronic kidney disease in diabetics and hypertensive patients

Citación recomendada:

Fuentes MartínezTR, Ríos NM, Argueta Coca JB. Conocimientos, actitudes y prácticas de prevención sobre la enfermedad renal crónica en diabéticos e hipertensos. Alerta. 2021;4(1):12-18. DOI 10.5377/alerta.v4i1.9538

Recibido:

12 de marzo de 2020

Aceptado:

12 de enero de 2021

Publicado:

22 de enero 2021

\section{Contribución de autoría:}

TRFM': Idea e implementación

de la investigación, NCR²

Aporte en la conducción de

la investigación, recolección de entrevistas y análisis de los resultados, JBAC ${ }^{3}$ : Conducción de la investigación, recolección de información a través de entrevistas y análisis de los resultados.

\section{Conflicto de intereses:}

Los autores no tienen conflicto de intereses.

\begin{abstract}
Resumen
Introducción. Según la Organización Mundial de la Salud, la cuarta parte de la población mundial sufre hipertensión arterial y diabetes mellitus tipo 2. Al padecer estas dos enfermedades, el riesgo de desarrollar enfermedad renal crónica, se multiplica. Objetivo. Analizar los conocimientos, actitudes y prácticas sobre la prevención de enfermedad renal crónica en usuarios con diabetes mellitus tipo 2 e hipertensión arterial, en el municipio de Jocoaitique, departamento de Morazán. Metodología. Estudio transversal descriptivo. Los datos fueron recolectados en visitas domiciliares y en la consulta general, realizando una entrevista a 125 personas, 108 hipertensos y 17 diabéticos. Luego fueron analizados a través del programa Perfect Statistics Professionally Presented versión libre. Resultados. De los participantes, el $96 \%$ tiene un bajo nivel educativo, el $4 \%$ ha realizado bachillerato y estudios superiores; el $83 \%$ pertenece al área rural y la ocupación predominante es ama de casa (58\%); el resto son jornaleros y agricultores (38\%). El $73 \%$ tienen un nivel medio de conocimientos, el $66 \%$ actitudes de indiferencia y el $76 \%$ realizan prácticas inadecuadas de autocuidado en la prevención de la enfermedad renal crónica. Conclusiones. Los conocimientos sobre la prevención de la enfermedad renal crónica, se encuentran en un nivel medio en la mayoría de los encuestados. Más de la mitad de la población expresa actitudes de indiferencia y más de la tercera parte realiza prácticas inadecuadas de prevención sobre la enfermedad renal crónica.

Palabras claves

Hipertensión, diabetes mellitus tipo 2, enfermedad renal crónica, conocimientos, actitudes y prácticas en salud.
\end{abstract}




\section{Introducción}

A nivel mundial los casos de enfermedades crónicas degenerativas van en aumento, lo que representa un problema importante para la salud pública'. Uno de los objetivos de la Organización Mundial de la Salud (OMS) es fomentar estilos de vida saludable en personas que padecen una o más enfermedades crónicas y que aprendan a convivir con esta patología ${ }^{2}$.

Según las estadísticas, entre las principales causas de mortalidad, a nivel nacional en el año 2019, estaban las enfermedades del sistema genitourinario, neumonías, enfermedades del sistema digestivo, septicemia, enfermedades del hígado, diabetes mellitus, enfermedades cerebro vasculares, enfermedades hipertensivas, otras enfermedades del corazón y el resto enfermedades del sistema respiratorio ${ }^{3}$.

En una encuesta realizada por el Instituto Nacional de Salud, se determinó que el $38 \%$ de las mujeres tienden a padecer hipertensión arterial (HTA), mientras que en los hombres el porcentaje es menor (35,8\%). En relación con la diabetes, los resultados de la encuesta reflejan que el 13,9\% de las mujeres y el 10,6\% de los hombres padecen la enfermedad ${ }^{4}$. Por otra parte, las enfermedades cardiovasculares, la enfermedad renal crónica (ERC), y el cáncer presentaron las tasas de mortalidad más elevadas en los hombres. En el caso de las mujeres, las enfermedades cardiovasculares, el cáncer y la ERC fueron las que presentaron las tasas de mortalidad más elevadas 5 .

De acuerdo con los datos del Sistema Único de Información en Salud (SUIS), en el año 2018, Morazán fue uno de los departamentos que presentó más casos de Enfermedades Crónicas no Transmisibles (ECnT). En primer lugar se encuentra la HTA, con 8978 personas afectadas, seguido por la diabetes mellitus 2 (DM2) con 23015. En el 2018 se registraron 81 casos con DM2 y 370 con HTA, en el municipio de Jocoaitique, departamento de Morazán'.

En el departamento de Morazán, no hay estudios realizados sobre conocimientos, actitudes y prácticas (CAP) sobre la prevención de la ERC y se desconoce el impacto que esta enfermedad tendría directamente en la calidad de vida de los pacientes con DM2 e HTA, que no tengan conocimientos, actitudes y prácticas adecuadas sobre el autocuido. En este contexto, se consideró necesario realizar un estudio sobre la prevención de la ERC en los pacientes con diagnóstico de DM2 e HTA. Por lo cual, el presente estudio tiene como objetivo analizar los conocimientos, actitudes y prácticas sobre la prevención de la enfermedad renal crónica en usuarios con diabetes mellitus tipo 2 o hipertensión arterial, en el municipio de Jocoaitique, departamento de Morazán.

\section{Metodología}

Se realizó un estudio cualitativo, transversal descriptivo, entre marzo y diciembre del año 2019, en el municipio de Jocoaitique del departamento de Morazán. Este municipio tiene dos Unidades Comunitarias de Salud Familiar (UCSF): una intermedia y una básica. Se eligió la UCSF Jocoaitique por ser un establecimiento intermedio y con mayor número de recursos humanos y más prestaciones de servicios, teniendo una población de estudio conformada por 183 personas, 29 personas con diagnóstico de DM2 y 154 con diagnóstico de HTA.

Para el cálculo de la muestra se aplicó el muestreo probabilístico estratificado, utilizando el programa Epidat versión 4,27,8. En el proceso se utilizó un nivel de confianza del $95 \%$, obteniendo los estratos para la muestra de la manera siguiente: para el estrato de pacientes con diagnóstico de DM2, que es de 29 personas, la muestra fue de 17. Para el estrato de pacientes con diagnóstico de HTA, que es de 154 personas, la muestra fue de 108, obteniendo un total de 125 personas.

La unidad de análisis fueron las personas con DM2 o HTA, a las que se les describieron las variables de conocimientos, actitudes y prácticas sobre la alimentación, ejercicio, consumo de tabaco, alcohol y control del tratamiento. Dichas variables fueron evaluadas según la escala de Likert? 9 .

Para la recolección de datos se utilizó un cuestionario estructurado con preguntas cerradas, conformado por tres partes: en la primera, el consentimiento informado; la segunda, datos de identificación, objetivo, instrucciones y aspectos socio demográficos; la tercera parte, tres apartados acerca de las variables CAP, enfocadas en la prevención de la ERC.

El instrumento de recolección de datos constó de 57 preguntas, con múltiples alternativas de respuesta. Se establecieron 16 preguntas acerca de los conocimientos sobre ERC, alimentación, ejercicio, ingesta de alcohol, tabaco y el control del tratamiento. El instrumento fue clasificado según escala de Likert en nivel de conocimiento bajo (0 a 19 puntos), nivel de conocimiento medio (19 a 29 puntos) y nivel de conocimiento alto (30 a 32 puntos). Asignando 1 punto al nivel bajo de conocimientos, 2 puntos al nivel de conocimientos medio y 3 puntos al nivel de conocimientos alto. 
Para las actitudes se establecieron 13 preguntas enfocadas en las mismas variables, clasificándolas según Likert como: actitud negativa ( 0 a 13 puntos), actitud indiferente (14 a 26 puntos) y actitud positiva (27 a 39 puntos) $)^{9}$. Para esta dimensión se asignó 1 punto a cada respuesta de actitud negativa, 2 puntos a las respuestas de actitud de indiferencia y 3 puntos a las respuestas de actitud positiva.

Finalmente, para describir las prácticas de prevención de la ERC se realizaron preguntas orientadas a la alimentación, ejercicio, ingesta de alcohol y/o tabaco y control de tratamiento. Se asignó 1 punto a cada respuesta de prácticas inadecuadas, 2 puntos a las prácticas adecuadas. Estas se clasificaron como inadecuadas de 1 a 13 puntos y adecuadas de 14 a 26 puntos $^{10}$.

Para la validación del instrumento de recolección de datos se ejecutó un plan piloto. Se seleccionaron a 10 usuarios con diagnósticos de DM2 o HTA de la UCSF de Yoloaiquín; una vez recolectados los datos, para obtener la confiabilidad de cada sección del cuestionario y validarlo, se aplicó Alfa de Cronbach, con el programa PSPP, que relaciona variables y establece la confiabilidad. El puntaje adquirido fue de 0,6, que por norma es inaceptable, por lo que se modificó y se realizó nuevamente la prueba piloto. En esta ocasión se seleccionaron a otras 10 personas con características similares a los sujetos en estudio, pertenecientes a la UCSFE San Francisco Gotera, del departamento Morazán. Posterior a la obtención de los datos se ejecutó el coeficiente Alfa de Cronbach, en PSPP, y se obtuvo un puntaje de 0,8. Este valor indica que el instrumento tiene una fiabilidad del $81 \%$, por lo que se admite como validado ${ }^{11}$

El estudio se inició en el mes de marzo y finalizó en diciembre del año 2019. La información fue recolectada por el personal del comité de investigación de enfermería del SIBASI Morazán. La recolección de la información se realizó en visitas casa a casa; también se entrevistaron a las personas que llegaron a consultar en horas hábiles a la UCSFI de Jocoaitique. Una vez concluida la recolección de la información, se digitó en el programa PSPP y luego se analizaron los datos obtenidos en cada variable. La elaboración de gráficos se realizó mediante el software Microsoft Excel 2010.

Previo a la ejecución de la investigación, se gestionó la autorización por parte de la directora de la UCSFI Jocoaitique Morazán y del Comité de Ética de la Región Oriental de Salud. Antes de la entrevista, se explicó a cada participante el propósito del estudio y se obtuvo la firma voluntaria del consentimiento informado.

\section{Resultados}

El $60 \%$ de la población estudiada son mujeres; el 50 \% tienen más de 60 años, mientras que el $34 \%$ oscila entre 50 a 59 años de edad. El $16 \%$ son personas adultas jóvenes de 20 a 49 años que adolecen de DM2 e HTA. La ocupación predominante es ama de casa $(58 \%)$, el resto son jornaleros y agricultores (38\%) y empleado formal e informal (4\%).

El $57 \%$ del total de la población tiene estudios de nivel primario, el $29 \%$ alcanzó el nivel secundario, un $10 \%$ no sabe leer ni escribir, el $2 \%$ estudió bachillerato y $2 \%$ tiene estudios superiores. El $83 \%$ de la población encuestada pertenece al área rural. El $58 \%$ tiene estado civil de acompañados, el $22 \%$ casados, un $4 \%$ solteros, el $4 \%$ viudos y un $12 \%$ divorciados.

El $73 \%$ de las personas tiene un nivel de conocimiento medio sobre la prevención de la ERC según se observa en la Figura 1. El $86 \%$ conoce sobre ERC, el tipo de alimentación que les está permitido consumir (86\%), la frecuencia del consumo de las comidas (90\%), la cantidad de agua que deben consumir (91\%) y el consumo de sal (91\%). En la importancia de la duración de los ejercicios (91\%), el $22 \%$ desconoce la frecuencia recomendada para ejercitarse. El $99 \%$ percibe la importancia de no hacer uso del tabaco y alcohol. El $28 \%$ tiene conocimiento sobre el medicamento, dosis y frecuencia para su actual enfermedad.

Según los resultados que se expresan en la Figura 1, relacionados con la actitud sobre la prevención de la ERC, en los alimentos que le están permitidos consumir, el $66 \%$ de las personas mostró actitud de indiferencia. En la frecuencia de las comidas, la actitud es de negatividad (49\%). En lo que respecta a la cantidad de agua que deben consumir, la actitud es de negatividad (82\%). En el consumo de azúcar, un 44\% tiene una actitud negativa, el $15 \%$ de indiferencia y el $41 \%$ tiene una actitud positiva. Por otra parte, en el consumo de sal, la actitud de negatividad es del $44 \%$. El $47 \%$ de las personas asumen una actitud negativa para la realización del ejercicio. El $88 \%$ de la población manifiesta una actitud positiva ante el consumo del tabaco; así mismo, el 99 \% tiene actitudes positivas frente al consumo de alcohol. Por otra parte, el $88 \%$ de las personas tiene actitud positiva en la importancia de conocer el medicamento que ingieren. La actitud para realización de exámenes (glucosa), en los pacientes con diabetes y la toma de la presión arterial en los pacientes hipertensos, 
la actitud es de negatividad (42\%) y de indiferencia (30\%). La actitud para asistir a los controles médicos es del $45 \%$ de negatividad y $15 \%$ de indiferencia.

Los resultados que se muestran en la Figura 1 están relacionados a las prácticas de prevención de la ERC. Así, el 76 \% realiza prácticas inadecuadas sobre la prevención de la ERC. El 88 \% expresa que ingiere todo tipo de alimentos; el $85 \%$ ingiere de una a dos comidas diarias. El $85 \%$ consume menos de dos litros de agua al día. El 85 \% usa salero en la mesa y el $86 \%$ no realiza ejercicios. El 95 \% no consume el medicamento, según la indicación médica.

Los resultados indican que el 73 \% de los pacientes con DM2 e HTA tiene un nivel medio de conocimientos sobre la prevención de la ERC (Figura 1). El $66 \%$ de los pacientes con DM2 e HTA tiene actitudes de indiferencia sobre prevención de la ERC (Figura 1). El 76 \% de los pacientes con DM2 e HTA tiene prácticas inadecuadas sobre la prevención de la ERC (Figura 1).

\section{Discusión}

Los participantes del estudio tienen un nivel medio de conocimientos, con actitudes de indiferencia y prácticas inadecuadas sobre la prevención de la ERC. Estos resultados son similares a los encontrados en Colombia ${ }^{12}$. Más de la mitad de la población es mayor de sesenta años, aumentando el riesgo de padecer la ERC. Estos resultados coinciden con los encontrados en un estudio realizado en Barcelona, en la atención primaria, donde se concluye que hay mayor probabilidad de desarrollar la ERC en la población adulta mayor, sobre todo si hay una enfermedad crónica ${ }^{13}$.

En cuanto al conocimiento en las variables alimentación, ejercicio, consumo de alcohol, tabaco y control del tratamiento, los datos describen que hay conocimientos en nivel medio según la escala de Likert. Los resultados son diferentes a los encontrados en un estudio realizado en Nicaragua, en el que concluyen que el conocimiento es alto pero distorsionado; por tanto, conlleva a las personas tener actitudes desfavorables de prevención ${ }^{14}$.

En la variable conocimiento, la mayoría de la población encuestada tiene conocimientos sobre ERC, el tipo de alimentación que les está permitida consumir, la frecuencia del consumo de las comidas, la cantidad de agua que deben ingerir, el consumo de sal y control del tratamiento. Esto indica que hay una buena educación de parte del personal del Ministerio de Salud (Minsal), según lo indicado por las Guías clínicas de medicina interna (2018). En el indicador sobre la realización de ejercicio para prevenir la ERC, los conocimientos son básicos. Por otra parte, en lo que respecta a la importancia del tiempo y frecuencia del ejercicio, las perso-

Figura 1: Niveles de conocimientos sobre la prevención de la enfermedad renal crónica.

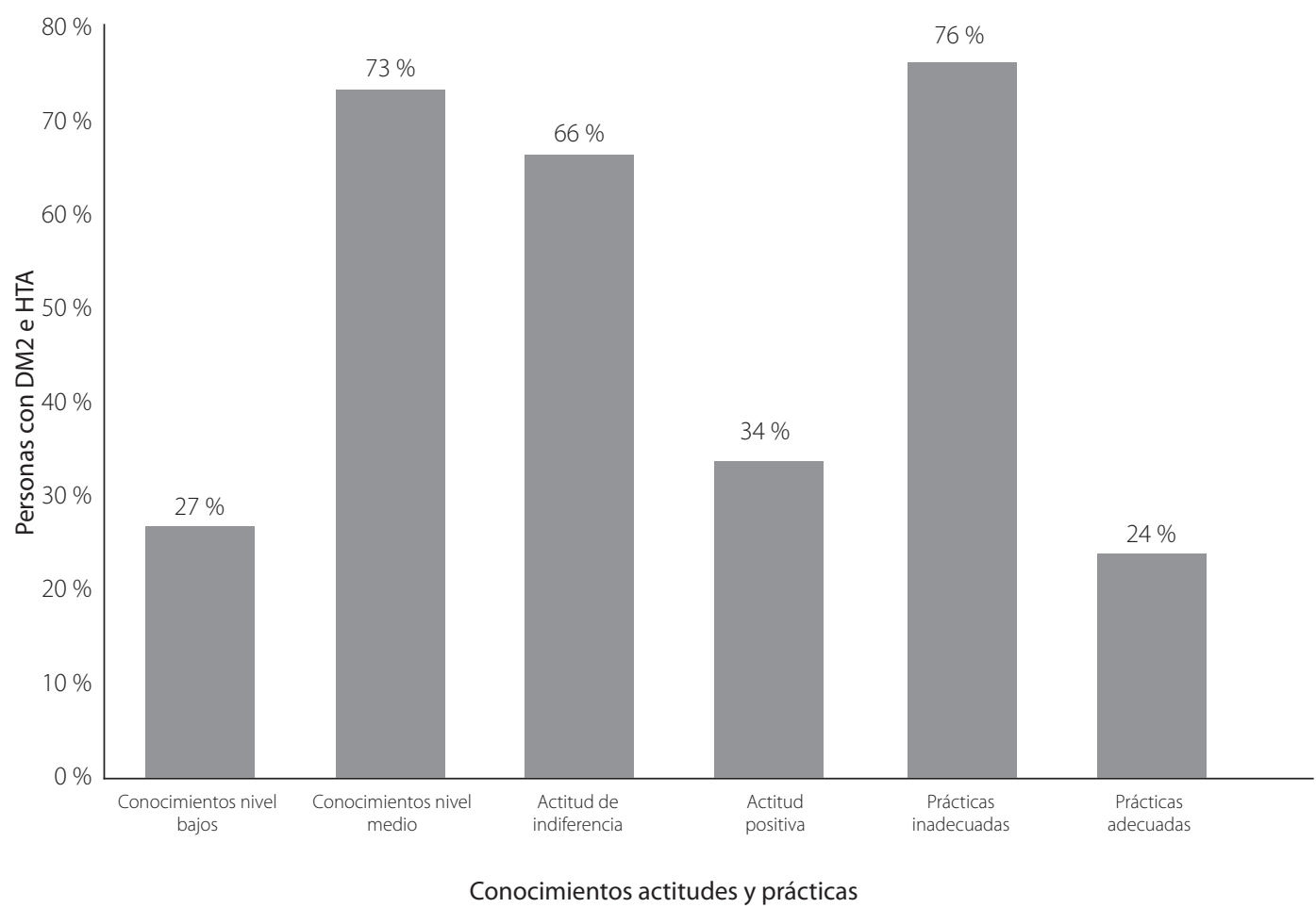


nas saben que es una medida de prevención para la HTA, DM2 y la ERC. El $99 \%$ de los entrevistados tiene los conocimientos necesarios sobre la importancia de no consumir tabaco y alcohol. Esto disminuye el riesgo de progresión a la ERC, a diferencia de los resultados en un estudio realizado en Cuba, donde el consumo de estas sustancias está en aumento ${ }^{15}$.

En la variable conocimiento del medicamento para la HTA y DM2, un cuarto de la población desconoce cuál es la dosis y la frecuencia del tratamiento que consume. Estos resultados son similares a los encontrados en una investigación realizada en Cuba, donde el desconocimiento del nombre del medicamento y la dosis indicada es una de las principales causas del abandono del tratamiento. Sin embargo, la adherencia es la base principal en el control de la enfermedad $^{16,17}$.

La actitud ante los alimentos que le están permitidos consumir, la frecuencia de estos, la cantidad de agua, el consumo de azúcar y sal, en más de la mitad de la población estudiada es de indiferencia ${ }^{17}$. En el indicador referente al ejercicio, más de un cuarto de la población tiene actitudes de negatividad, datos similares a los encontrados en un estudio realizado en Honduras ${ }^{18}$. Así mismo, el control del tratamiento, en tres cuartos de la población, es de negatividad. En consecuencia, en este mismo apartado, un tercio de la población tiene actitudes de indiferencia frente a la realización de exámenes y la toma de presión arterial. En un estudio realizado en Cuba, se concluye en que una persona cambiará su estilo de vida para generar cambios si es capaz de percibir los riesgos a los que está expuestos ${ }^{18}$.

En relación con las prácticas, la mayoría de las personas expresó que realizan prácticas inadecuadas, entre ellas que ingieren todo tipo de alimentos, realizan de una a dos comidas diarias, consumen menos de dos litros de agua al día, el uso del salero de mesa es frecuente; en más de tres cuartos de la población no realizan ningún tipo de ejercicios. Estos resultados son similares a los encontrados en un estudio en Rawalpindi, donde el conocimiento de los pacientes en estudio fue adecuado y las prácticas eran deficientes ${ }^{19}$.

En el consumo de alcohol y tabaco las prácticas son adecuadas. Estos resultados pueden deberse a que más de la mitad de la población estudiada eran mujeres. Por otra parte, menos de una décima parte de la población en estudio consume tabaco e ingiere alcohol, similar a un estudio realizado en México, en el que los resultados obtenidos muestran que los hombres que padecen de una enfermedad crónica, como DM2 e HTA, más otras enfermedades, consumían tabaco y alcohol con frecuencia ${ }^{20}$. En consecuencia, el medicamento no es ingerido según las indicaciones médicas; de igual forma, los exámenes no se realizan en las fechas indicadas por la mayor parte de la población en estudio.

Los resultados reflejan que la ocupación predominante en más de la mitad de la población es ama de casa y un tercio de la población tiene ocupación de jornalero y agricultor. Con respecto al nivel educativo, una décima parte de la población es analfabeta y más de la mitad tienen un nivel académico de primaria, ya sea esta completa o incompleta y un mínimo porcentaje alcanzó la educación superior (técnica o universitaria). El bajo nivel de escolaridad, por lo general, propicia que las personas no acaten las recomendaciones médicas.

La mayoría de los participantes pertenecen al área rural, lo que dificulta la accesibilidad a los servicios de salud más cercanos y al control médico. Estos resultados son similares a un estudio realizado en Perú, en el que pertenecer al área rural dificulta el acceso a los servicios de salud ${ }^{21}$. Dorothea Orem, en su teoría del autocuidado, menciona que autocuidarse es una función humana y reguladora; debe realizarse de forma continua e ir enfocada al autocuido para la prevención de enfermedades. En este sentido, las prácticas sobre la prevención de la ERC difieren de la teoría de Dorothea Orem²2. El incremento de la ERC en personas con HTA y DM2, es un riesgo; en el estudio se encontraron conocimientos en un nivel medio. Estos resultados coinciden con los encontrados en un estudio realizado en Madrid, en el que la población estudiada no realiza prácticas de prevención enfocadas a disminuir el riesgo de ERC 23

De acuerdo con los resultados encontrados, es preciso enfocar actividades de información, educación y comunicación, en las personas, con DM2 e HTA ${ }^{24}$, identificadas con déficit de conocimientos, actitudes de indiferencia y prácticas de autocuidado inadecuadas, e implementar planes de autocuidado integral, para mejorar la calidad de vida y propiciar los estilos de vida saludable $^{25}$. No obstante, sería beneficioso el abordaje psicológico, en los grupos con factores de riesgo, de desarrollar la ERC, enfocados a los cambios de actitud. El total de personas con enfermedades crónicas como la DM2 e HTA deberían tener atención integral a cargo de personal multidisciplinario de forma continua, a fin de mejorar las actitudes y, por ende, las prácticas de prevención de la enfermedad renal crónica ${ }^{26,27}$. 


\section{Conclusión}

El nivel de conocimientos sobre la prevención de la enfermedad renal crónica que tienen los pacientes con diagnóstico de DM2 e HTA, según la escala de Likert, se encuentra en un nivel medio en la mayoría de los usuarios encuestados.

En cuanto a las actitudes, son de indiferencia en más de la mitad de la población. Debido a ello, en este estudio se evidencia que las prácticas de autocuidado sobre la enfermedad renal crónica en pacientes con DM2 e HTA son negativas. Más de la tercera parte de la población encuestada no está realizando las prácticas adecuadas en la prevención de la ERC.

\section{Agradecimiento}

A la maestra Meiby Sulema Rivera Vásquez, por brindarnos su apoyo técnico en el proceso. A Licda. Reina Isabel Campos de Santin, por su apoyo en durante el proceso de publicación.

\section{Financiamiento}

Fuentes propias de los autores del estudio

\section{Referencias bibliográficas}

1. Organización Panamericana de la Salud. Plan de acción para la prevención y el control de las enfermedades no transmisibles en las Américas 2013-2019. Washington, D.C.: OPS. 2014. Fecha de la consulta: 12 de mayo 2019. Disponible en: https://iris.paho.org/ handle/10665.2/35010

2. Valerie A, Marcello T, John S. La carga global de la insuficiencia renal y los objetivos de desarrollo sostenible. Organización Mundial de la Salud. 2018. Fecha de consulta: 6 de junio 2019. Disponible en: https://www. who.int/bulletin/volumes/96/6/17-206441ab/es/

3. Ministerio de Salud Pública. Estadísticas de Morbi-Mortalidad 2019. Fecha de la consulta: 13 de diciembre de 2020. Disponible en: https://simmow.salud.gob.sv

4. Instituto Nacional de Salud. Encuesta Nacional de Enfermedades Crónicas no Transmisibles en Población Adulta de El Salvador, ENECA-ELS 2015. San Salvador, El Salvador, 2017. 252 p.

5. Instituto Nacional de Salud. Carga de mortalidad de enfermedades no transmisibles en la población igual o mayor de 20 años de El Salvador. San Salvador. 2017. 44p. Disponible en: $\underline{\text { http://ins.salud. }}$ gob.sv/wp-content/uploads/2018/07/
Carga-de-mortalidad-de-enfermedades-no$\underline{\text { transmisibles }}$

6. Ministerio de Salud. Sistema de Información de Ficha Familiar. Fecha de consulta: 11 de mayo de 2019. Disponible en: https://siff. salud.gob.sv/

7. Otzen T, Manterola C. Técnicas de muestreo sobre una población a estudio. Revista International Journal of Morphology. 2017; 35 (1): 227-232. DOl: http://dx.doi. org/10.4067/S0717-95022017000100037

8. Hernández C, Carpio N. Introducción a los tipos de muestreo. Revista Alerta. 2019; 2(1): 75-79. DOI: https://doi.org/10.5377/alerta. v2i1.7535

9. García S, Aguilera J, Castillo A. Guía técnica para la construcción de escalas de actitud. Revista electrónica de pedagogía.

2011;8(16): 1-13. Disponible en: http://www. odiseo.com.mx/2011/8-16/pdf/garciaaguilera-castillo-guia-construccion-escalasactitud.pdf

10. Halleslevens A, Gutiérrez D. Conocimientos, actitudes y prácticas sobre la alimentación adecuada en los pacientes que son sometidos a hemodiálisis en HEODRA-León en el período de marzo a junio del 2018. Tesis de medicina y cirugía. Nicaragua. Universidad Nacional Autónoma de Nicaragua, León Sistemas de Biblioteca; 2019. $46 \mathrm{p}$.

11. Corral Y. Validez y confiabilidad de los instrumentos de investigación para la recolección de datos. Revista Ciencias de la Educación. 2009;19 (33): 229-247. Disponible en: https://www.calameo.com/ books/00441616680da9a5cd6ab

12. Ramírez C, Perdomo A, Rodríguez M. Conocimientos y prácticas para la prevención del pie diabético. Revista Gaúcha Enferm. 2019; 40:e20180161. DOI: https://doi.org/10.1590/19831447.2019.20180161

13. Salvador $B$, Rodríguez $M$, Ruipérez $L$, Ferré A, Canillera O, Rodríguez L. Enfermedad renal crónica en individuos hipertensos $\geq 60$ años atendidos en Atención Primaria. Revista Nefrología. 2017; 47(4):236-45. DOl: 10.1016/j.nefro.2017.02.008

14. Ballesteros A, Kulakova O, Jirón A, Martínez J, Urcuyo N. Conocimiento, actitudes y prácticas sobre enfermedad renal crónica en habitantes de la comunidad «La Isla» del municipio de Chichigalpa. Revista Científica UNAN-León. 2013; 4 (1) 53-57. DOl:https:// doi.org/10.5377/universitas.v4i1.1674

15. Landrove O, Morejón A, Venero S, Suárez R, Almaguer M, Pallarols E. Enfermedades no transmisibles: factores de riesgo y acciones para su prevención y control en Cuba. Revista Panamericana Salud Pública. 2018; 42: e23. DOI: https://doi.org/10.26633/ RPSP.2018.23 
16. Achiong F, González Y, Vega O, Guillot O, Rodríguez A, Díaz A. Algunos resultados postintervención en el control, tratamiento y complicaciones de la hipertensión arterial. Policlínico Universitario Héroes del Moncada. Cárdenas. Revista Médica Electrónica. 2016; 38(4): 553-64. Disponible en: http://scielo.sld.cu/scielo. php? script $=$ sci arttext\&pid=S1684$18242016000400005 \& \operatorname{lng}=\mathrm{es}$.

17. Miranda V, Mejía D, Paredes F, López D, Romero J. Conocimientos, actitudes y prácticas de pacientes hipertensos en San José de Boquerón. Revista Científica de la Escuela Universitaria de las Ciencias de la Salud. 2018; 6(1):21-28. DOI: https://doi. org/10.5377/rceucs.v6i1.8396

18. Gonzales M, Pérez G. Conocimientos, creencias y prácticas en pacientes hipertensos, relacionados con su adherencia terapéutica. Revista Cubana de enfermería. 2005;21 (15):1-8. Disponible en: http://scielo. sld.cu/pdf/enf/v21n3/enf08305.pdf

19. Tahir N, Imran M, Hashmi A, Zeb A, Furqan A. Knowledge, Attitudes And Practices Of Patients Regarding Diabetes And Hypertension Control. J Postgrad Med Inst. 2018;32(2):166-72. Disponible en: https://www.researchgate.net/ publication/329672647

20. Pavón P, Gogeascoechea M, Blázquez M, Sánchez A. Factores asociados con el consumo de alcohol y tabaco en adultos mayores. Revista Médica de la Universidad Veracruzana. 2020;18(1):7-16. Disponible en: https://www.medigraphic.com/pdfs/ veracruzana/muv-2018/muv181b.pdf

21. Azañedo D, Vargas R, Rojas C. Factores asociados al uso de servicios de salud oral en adultos mayores peruanos: análisis secundario de encuesta poblacional. Revista Perú Med Exp Salud Pública. 2018; 36:553-61. DOl:https://doi.org/10.17843/ rpmesp.2019.364.4724
22. 22. Naranjo Y, Concepción J, Rodríguez M. La teoría Déficit de autocuidado: Dorothea Elizabeth Orem. Revista SciELO Analytics. 2017;19(3):1-11. Disponible en: http://scielo. sld.cu/scielo.php?script=sci arttext\&pid $=$ S1608-89212017000300009

23. Ruiz J, Ríos A, Rodríguez J, Llorente S. Prevalencia y riesgo de progresión de enfermedad renal crónica en pacientes diabéticos e hipertensos seguidos en atención primaria en la Comunidad de Madrid. Revista de Nefrología. 2017;37(3):338-354. DOl: https://doi. org/10.1016/..nefro.2016.10.019

24. Costa G, Moreso F, Cantarell M, Cerón D. Alfabetización en salud y enfermedad renal crónica. Revista de la Sociedad Española de Nefrología. 2017;37(2):115-117. DOI: http:// dx.doi.org/10.1016/j.nefro.2016.10.001

25. Pinillos Y, Herazo Y, Gil J, Ramos J. Actividad física y calidad de vida en personas con enfermedad renal crónica. Revista médica Chile. 2019;147(2): 153-160.

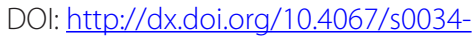
$\underline{98872019000200153}$

26. Landazábal A, Perea D, Garizabalo O, Sanabria M, Vesga J, Roderos I, Muños F, Laganis F, Lopéz P. Programa de prevención de la enfermedad renal crónica basado en redes integradas de servicios en Colombia. Revista salud pública. 2017;19 (2). DOI: https://doi.org/10.15446/rsap. v19n2.45110

27. San Martin V, Caballero A, Camacho C, Giménez B, San Martin V, Caballero A, et al. La Extensión Universitaria como herramienta para mejorar conocimientos de pacientes con diabetes e hipertensión. An Fac Cienc Médicas Asunción. 2018; 51(3): 69-74. Disponible en: http://dx.doi. org/10.18004/anales/2018.051(03)69-074 